\title{
Transmission du Trypanosoma cruzi chez les Mammifères
}

\author{
Par Carlos DIAZ-UNGRIA
}

\section{I. - Contamination par voie buccale à partir des excréments de RHODNIUS PROLIXUS infestés}

Dans ces dernières années, les études sur Trypanosoma cruzi ont pris une importance croissante. Il est, en effet, chez l'homme l'agent de la maladie de Chagas incurable tant dans sa forme aiguë mortelle que dans sa forme chronique cardiopathique. Pour donner une idée de son importance au Vénezuela, rappelons que selon les différents auteurs, on estime qu'il y a entre 500.000 et 1.000 .000 de sujets atteints pour une population totale d'environ 6.000 .000 d'habitants. Pour nous, il est encore plus grave de savoir qu'à l'heure actuelle, dans des milliers de « ranchos » de l'Amérique tropicale et sub-tropicale, vivent des enfants exposés à une contagion qui risque d'en faire des malades pour le restant de leur vie.

Devant cette situation, il était naturel que les études de la maladie de Chagas humaine aient été en premier des enquêtes épidémiologiques ayant pour but de déterminer avec exactitude l'aire d'extension géographique de l'affection. L'urgence de ce travail préliminaire a fait quelque peu perdre de vue aux chercheurs américains le problème si important du mécanisme de la transmission. Celui-ci présente cependant aujourd'hui encore des points obscurs dont il apparaît que certains pourraient ne pas s'accorder exactement avec les conceptions classiques.

Pour les mêmes raisons, l'importance de la maladie humaine a laissé dans l'ombre le problème posé aux vétérinaires par l'existence de nombreux chiens porteurs du trypanosome. Ces animaux sont seulement considérés comme des réservoirs de virus pour l'homme alors, qu'en fait, ils présentent une maladie absolument similaire à celle de l'homme.

En notre qualité de vétérinaire, nous nous sommes attaché à l'étude de la maladie de Chagas canine et, tout particulièrement, au mode de transmission du trypanosome au chien. Nous espérions que, chemin faisant, notre étude pourrait élucider certaines énigmes qui subsistent dans le mécanisme de la transmission de la maladie à l'homme. Le chien paraît être le Mammifère qui paie le plus lourd tribut à la maladie de Chagas. 
Selon Pifano, au Venezuela, de 50 à $70 \%$ des chiens sont infestés dans les régions d'endémicité, chiffres qui sont très largement supérieurs au pourcentage des infestations humaines. Il apparaît que ce fait n'a pas été, jusqu'ici, suffisamment pris en considération dans l'organisation de la lutte contre la maladie humaine.

\section{Mécanisme de contamination}

La source de la contamination de l'homme et du chien est constituée par les déjections des «chipos» (Rhodnius prolixus) infestés par Trypanosoma cruzi. Il est établi que l'Insecte puise le trypanosome dans le sang' d'un Mammifère infesté (homme, chien, chat, rat, etc.). Après un processus d'involution, de multiplication suivie d'une nouvelle évolution, les trypanosomes métacycliques infestants apparaissent dans les excréments de l'Insecte. Ces formes assurent la contamination des Mammifères sains par diverses voies et c'est ce mode de pénétration du parasite dans l'organisme qui fait l'objet du présent travail.

Le milieu dans lequel se déroule le cycle évolutif du trypanosome et de son vecteur est l'habitation humaine, le « rancho » avec ses murs de terre et, surtout, son toit de palmes. Nous savons aujourd'hui, grâce au travail de Gamboa (1962) que les $R$. prolixus vivent hors de l'habitation humaine et spécialement dans les palmeraies où on les trouve déjà porteurs de trypanosomes. Ceux-ci proviennent sans doute du sang des animaux sauvages, des rongeurs ou des chiens, etc. C'est l'homme lui-même qui, utilisant les palmes pour couvrir son « rancho », y introduit le trypanosome et son vecteur. Nous pouvons déduire de ces observations que le chien peut s'infester, soit dans l'habitation de son maître, soit hors de celle-ci lorsqu'il dort dans la palmeraie. D'autres $R$. prolixus, cette fois non infestés, peuvent provenir d'un endroit différent, mais également extérieur à l'habitation: les nids des oiseaux sauvages et les poulaillers.

Une fois qu'il s'est établi dans l'habitation de l'homme, le $R$. prolixus infesté assure la transmission du trypanosome à l'homme, au chien, au chat, aux rongeurs, etc.

Selon les études faites chez l'homme, les voies de contamination acceptées sont les suivantes: voie oculaire, voie cutanée, voie transplacentaire et voie sanguine dans le cas de transfusion de sang d'un donneur infesté. On admet d'autres portes d'entrée plus rares : contamination de l'enfant par le lait de la mère, contamination par voie buccale pendant l'alimentation, contamination par voie vaginale et contamination accidentelle de laboratoire. De toutes ces voies, seules les deux premières sont considérées comme d'importance réelle dans les conditions naturelles de transmission.

La pénétration par voie oculaire transconjonctivale a toujours été considérée comme le mode de contamination habituel de l'homme. Elle se fait pendant la nuit, lorsque le $R$. prolixus se gorge sur la joue du dormeur et y dépose, peu après, ses excréments. Les auteurs affirment que c'est l'homme lui-même qui, pendant son sommeil ou au réveil, en posant sa main sur sa joue, y récolte les excréments frais de l'insecte et les dépose sur ses yeux. Chez l'homme ce mode d'inoculation produit une lésion de pénétration pathognomonique qui porte le nom de complexe ophtalmo-gan- 
glionnaire ou signe de Romaña et consiste en un volumineux cedème palpébral unilatéral.

Dans le cas de la pénétration cutanée, il existe un chancre d'inoculation appelé chagome qui est un nodule inflammatoire ou une plaque infiltrée de type érysipélatoïde avec une aire centrale nécrotique et une adénopathie satellite. Nombre d'auteurs font remarquer qu'il est hautement improbable que les trypanosomes puissent parvenir à traverser la peau humaine. Ceci nécessite, en effet, de nombreuses circonstances favorables coexistantes qui ne se rencontrent guère que sur la peau délicate des enfants, lorsque les excréments des «chipos» sont encore humides et qu'il existe enfin des érosions qui permettent la pénétration des trypanosomes. Il est certain, ainsi que le démontre l'apparition du chagome, qu'il existe des cas de pénétration cutanée, mais ils sont probablement très rares. Il ne reste donc, comme voie fréquente de contamination actuellement reconnue, que la voie oculaire transconjonctivale. Selon Romaña, en Argentine, le syndrome oculaire s'observe dans $91,4 \%$ des cas, le chagome dans $3,2 \%$ et il reste $2,3 \%$ de cas dans lesquels la porte d'entrée demeure inconnue. Au Vènezuéla, Pifano (1960), observe le syndrome ophtalmo-ganglionnaire dans 22,22\% des cas, le chagome dans $6,66 \%$ alors qu'il reste $71,2 \%$ des cas vénézuéliens pour lesquels on peut discuter de la porte d'entrée du trypanosome. Si l'on essaie de transposer ces faits au chien, il devient évident que le mécanisme de pénétration oculaire ne peut jouer comme chez l'homme. En outre, sa peau est beaucoup plus difficile à traverser que la peau humaine. Il s'ensuit que le mécanisme d'inoculation doit être différent. Il en est de même pour le tatou et pour les autres animaux sauvages, rongeurs en particulier chez lesquels les voies de contamination oculaire et cutanée sont improbables.

De toute façon, il est nécessaire d'admettre que les Mammifères peuvent s'inoculer par grattage. Neveu-Lemaire cite le travail de Cardoso (1938) qui a réussi dans $100 \%$ des cas la transmission expérimentale par scarification cutanée. Récemment, au Venezuèla, Juan-C. Gomez-Nuñez, dans une communication verbale, nous a révélé avoir obtenu $100 \%$ de positivité de l'examen à frais du sang périphérique au bout de vingt jours, en utilisant la scarification cutanée chez la souris en tant que méthode de contrôle pour les excréments frais de $R$. prolixus. On peut donc penser que, d'après ces constatations, il est possible que le chien et les autres Mammifères s'inoculent par grattage, mais il n'est pas obligatoire d'admettre que c'est là ce qui se produit dans tous les cas.

\section{Travaux antérieurs sur la transmission par voie buccale}

En tenant compte des analogies qui existent entre $T$. cruzi et $T$. lewisi qui sont les seuls trypanosomes qui se transmettent par les excréments des Invertébrés vecteurs, et en se souvenant que les rats s'infestent par $T$. lewisi en dévorant leurs puces, il était logique de penser que les chiens pouvaient s'infester par $T$. cruzi en dévorant, soit l'Invertébré vecteur correspondant ( $R$. prolixus), soit ses déjections. Déjà, en 1931, Emile Brumpt infectait expérimentalement un agneau de trois semaines en déposant sur la 
muqueuse buccale les excréments de Triatomes infestés. Talice (1944) suppose que le chien peut s'infester par voie buccale en écrasant dans sa gueule les Triatomes et il inclut la voie orale, pendant l'alimentation, comme mode possible de contamination de l'homme. Torrico (1950) relate avoir observé comment le cobaye dévore les Triatomes infestés et constaté, huit jours plus tard, la présence de trypanosomes dans son sang. Romaña (1963) cite une observation de Dias qui a vu un chat de « rancho * manger des Triatomes.

C'est Mayer (1961) qui apporte enfin la preuve expérimentale définitive en alimentant des rats, des souris, des chiens et des chats avec du lait contenant, pour $20 \mathrm{~cm}^{3}$, une goutte d'excréments de $T$. infestans contenant $T$. cruzi. Dans cette expérience unique, les résultats obtenus ont été les suivants: l'examen à frais du sang périphérique montra que toutes les souris étaient positives après un délai d'incubation de 11 à 24 jours, le chat de deux mois se montra constamment positif après une incubation de 22 à 27 jours, le chien d'un à deux mois se montra constamment positif après une incubation de 14 à 29 jours, les jeunes rats se montrèrent positifs après une incubation de 9 à 25 jours, quatre par l'examen à frais du sang, un par le xénodiagnostic, les rats adultes enfin se montrèrent dans $50 \%$ des cas négatifs et dans $50 \%$ des cas positifs uniquement par le xénodiagnostic.

\section{Expériences réalisées par nous}

Au départ, nous pensions que des expériences de Mayer pouvaient découler bien des conséquences du plus haut intérêt en ce qui concerne la connaissance de la transmission de la maladie de Chagas au chien et peut-être à l'homme. La première partie de notre travail a consisté à refaire l'expérimentation de Mayer pour vérifier si ses résultats étaient identiques au Vènezuela où existe $R$. prolixus en lieu et place du $T$. infestans qu'à utilisé l'auteur argentin.

Nos expériences qui ont débuté en février 1964 sont les suivantes :

$1^{\circ}$ Notre ami, le Docteur Manuel Dominguez Querada, nous a donné un lot de $\boldsymbol{R}$. prolixus infestés à $70 \%$ presque exclusivement par $T$. cruzi.

$2^{\circ}$ Nous avons isolé les Rhodnius infestés par $T$. cruzi. Leurs abdomens ont été écrasés et les excréments ainsi obtenus mélangés à de l'eau physiologique. Ce broyat a été ajouté à du lait dans une proportion que nous n'avons pas déterminée et ce liquide administré à des lots d'animaux différents.

$3^{\circ}$ Sept souris sont nourries individuellement grâce à une pipette introduite dans la gueule. Les animaux ont avalé avec difficulté le liquide et l'on peut considérer que la quantité réellement ingérée fut très réduite.

$4^{\circ}$ Dix rats adultes furent isolés dans une seule cage et reçurent, comme boisson, le mélange de lait et d'excréments positifs disposé dans un biberon.

Nous pensons que tous les rats ont bu ce liquide encore que, ne les ayant pas observés sans arrêt, il nous est impossible de l'affirmer. 
$5^{\circ}$ Cinq cobayes ont reçu une dose individuelle au moyen d'une pipette et ont bu de façon suffisante.

$6^{\circ}$ Sept chiens âgés de cinq semaines reçoivent le mélange, chaque chien ayant son écuelle individuelle contenant le lait contaminé. Tous ont bu facilement. Nous avons préalablement vérifié que les chiens n'étaient pas infestés par $T$. cruzi (examen du sang périphérique).

$7^{\circ}$ Une chienne adulte, mère de quelques-uns des chiots du lot précédent, ingère du lait contaminé sans difficulté.

Les résultats de ces expériences sont les suivants :

- Les sept chiots sont positifs à l'examen à frais du sang périphérique après une incubation de 16 jours pour deux d'entre eux, 19 jours pour quatre et 20 jours pour un.

- Quatre cobayes sur cinq sont positifs à l'examen à frais du sang périphérique après une incubation de 21 jours pour deux d'entre eux et 22 jours pour les deux autres. Le dernier est malade pour une cause inconnue et 28 jours après la contamination, immédiatement avant sa mort, nous pratiquons un xénodiagnostic qui est positif 38 jours plus tard.

- Six rats adultes sur dix sont positifs à l'examen à frais du sang périphérique après une incubation de 22 jours pour deux d'entre eux, 25 jours pour un, 29 jours pour deux et 33 jours pour un. Pour les quatre autres on pratique, avant de les sacrifier, un xénodiagnostic au $33^{\circ}$ jour. Deux seront positifs 41 jours plus tard et deux resteroat négatifs.

- Aucune des sept souris ne présente de trypanosomes dans le sang périphérique, mais les xénodiagnostics pratiqués au $47^{\circ}$ jour de la contamination se montreront tous positifs 41 jiours plus tard. Juan C. Gomez-Nuñez nous a dit avoir obtenu des résultats identiques ( $60 \%$ de positivité) en administrant par voie buccale, à la souris, des $R$. prolixus infestés et leurs excréments. En utilisant des Insectes infestés morts depuis trois jours, $20 \%$ des souris ont été positives à l'examen à frais du sang périphérique après une incubation de 27 jours.

En outre, ajoutons que l'un des chiots qui présentait des trypanosomes sur les frottis de sang, lesquels avaient tous les caractères morphologiques de $T$. cruzi a été sacrifié un mois et demi après la contamination. A l'autopsie, nous avons trouvé des lésions typiques de myocardite chagasique aiguë avec présence d'amas de leishmanies. Chez l'un des rats dont le sang était positif, nous avons également observé un envahissement massif du cœur par des leishmanies, 22 jours après la contamination buccale.

\section{Conclusions}

De ces expériences, Mayer concluait que, dans le « rancho », les Triatomides laissent tomber leurs déjections du haut du toit sur les biberons, les verres, les tables et les ustensiles de cuisine, ce qui rend possible la contamination de l'homme par 
TABLEAU I

Infection par voie orale. Incubation en jours

\begin{tabular}{|c|c|c|c|c|c|c|c|c|c|}
\hline & \multicolumn{8}{|c|}{ Examen sur le sang frais } & \multirow{2}{*}{ Xénodiagnostic } \\
\hline & 16 & 19 & 20 & 21 & 22 & 25 & 29 & 33 & \\
\hline 7 souris . & - & - & - & - & - & - & - & - & $\begin{array}{l}7 \text { xéno. }\left(47^{\circ} \text { jour }\right) \\
\text { tous }+(41 \mathrm{j} . \text { après })\end{array}$ \\
\hline 10 rats $\ldots$ & - & - & - & - & $2+$ & $1+$ & $2+$ & $1+$ & $\begin{array}{l}4 \text { xéno. }\left(33^{\circ} \text { jour }\right) \\
2+(41 \text { jours après }) \\
2-\end{array}$ \\
\hline 5 cobayes. & - & - & - & $2+$ & $2+$ & & & & $\begin{array}{l}1 \text { xéno. }(28 \text { jour }) \\
+(38 \text { jours après })\end{array}$ \\
\hline $\begin{array}{l}7 \text { chiens } \\
5 \text { semaines }\end{array}$ & $2+$ & $4+$ & $1+$ & & & & & & \\
\hline $\begin{array}{l}1 \text { chienne } \\
\text { adulte } \ldots\end{array}$ & - & - & - & - & - & - & - & $1+$ & \\
\hline
\end{tabular}

voie digestive. Celui-ci peut également se contaminer en portant à sa bouche ses doigts souillés par les excréments des punaises. Chez le chien la contamination se fait, soit par ingestion d'aliments souillés, soit par léchage et déglutition des déjections des Triatomides déposées sur son pelage.

Puisque dans leur ensemble nos résultats coïncident avec ceux de cet auteur, nos conclusions seront fatalement analogues. Il est certain que la voie buccale est le " mode habituel s de contamination du chien. Chez l'homme, on peut affirmer que la voie buccale est le mode de contamination dans tous les cas où il n'existe pas de témoin d'une porte d'entrée oculaire ou cutanée. De toute façon, les modalités de cette contamination peuvent être discutées et le but de la suite de notre expérimentation fut de tenter d'en élucider la nature.

\section{$* *$ \\ II. - Voie suivie par les Trypanosomes chez le Mammifère contaminé par la bouche}

Chez les Mammifères, les voies de pénétration du $T$. cruzi sont naturellement multiples, mais nous considérons que ce sont les voies oculaire, buccale et cutanée qui sont les plus fréquentes.

Chez l'homme, la voie oculaire est considérée comme la plus fréquente. Les faits expérimentaux, en particulier chez le singe, sont en faveur de cette affirmation : l'instil- 
lation de trypanosomes dans l'œil provoque le syndrome ophtalmo-ganglionnaire, puis la maladie.

Mais il importe de se souvenir qu'en règle générale les trypanosomes présentent un tropisme pour l'œil, plusieurs d'entre eux produisant même des lésions oculaires sans que leur pénétration dans l'organisme se fasse par cette voie. Ainsi dans le «souma » des Indes, provoqué chez les chevaux par T. evansi, on signale des lésions oculaires avec ecchymoses de la conjonctive. Chez le chien, en Inde, le même trypanosome provoque une conjonctivite avec opacité de la cornée. Au Vènezuela, $T$. venezuelense provoque chez le chien une maladie dans laquelle on observe une blépharite, une conjonctivite et une kératite. Dans le «mal de caderas» des chevaux du Brésil, du Paraguay et de l'Argentine dont l'agent est la variété américaine de $T$. evansi, il existe une conjonctivite avec ecchymoses oculaires en plus de la kératite interstitielle et de l'iritis. Dans la «derrengadera » des chevaux du Venezuela, due à $T$. venezuelense, on observe des lésions oculaires identiques. Dans la dourine des chevaux, transmise par le coït, existe un œdème et une ulcération de la cornée. Dans la trypanosomiase bovine à $T$. vivax, tant en Afrique qu'en Amérique, il y a une hypersécrétion lacrymale. Dans le nagana provoqué chez les animaux domestiques par $T$. brucei, existe un œdème oculaire avec opacité de la cornée et cécité. Dans la maladie du sommeil humaine à $T$. gambiense on observe un œdème sus-orbitaire, une irido-cyclite et une choriorétinite. Dans la forme bien plus sévère produite par $T$. rhodesiense, lequel détermine souvent une myocardite et une péricardite exsudative, car il se multiplie dans le myocarde, on a signalé que l'inoculation au mouton et à la chèvre entraîne un œdème oculaire et une kératite.

Rappelons, en outre, que selon divers auteurs, il existe, en plus de l'œdème palpébral d'origine exogène attribué à la contamination par voie oculaire à partir des déjections des réduves, un syndrome analogue, mais d'origine endogène. Il survient à quelque temps de la contamination et peut s'observer même dans les cas où la voie de pénétration n'a pas été oculaire (Talice, 1964). Bien que ce syndrome endogène soit avant tout un œdème facial qui n'intéresse que la paupière inférieure, il est bon de se souvenir de son existence lorsque l'on discute de la porte d'entrée.

En outre, chez le chien et les autres Mammifères, il ne semble pas que la voie oculaire soit fréquente car les circonstances nécessaires à ce mode de contamination tel qu'on les décrit pour l'homme (doigts souillés par les déjections virulentes de l'Insecte) ne peuvent être réunies. Nous pensons que chez le chien et les autres Mammifères, la voie buccale est le mode de contamination le plus fréquent. Dans un travail antérieur nous avons montré la facilité avec laquelle ceci peut être expérimentalement reproduit. Les Mammifères peuvent se contaminer, soit en léchant des objets, soit en ingérant des aliments souillés par les déjections virulentes de $R$. prolixus, soit, enfin, en dévorant les Insectes eux-mêmes, mais cette dernière éventualité nous semble rare.

La contamination par voie transcutanée peut être considérée comme la troisième par ordre d'importance chez l'homme et comme la seconde chez le chien et les autres Mammifères. En effet, les épreuves d'infestation par scarification cutanée sont toujours positives et l'on peut admettre que les animaux se contaminent par grattage. 
Puisque nous avions été amené à penser que le chien se contamine naturellement surtout par ingestion des déjections des Insectes, nous nous sommes prōposé d'étudier le cheminement du $T$. cruzi chez le chien infesté par cette voie.

Il importait de considérer en premier lieu la durée de survie des trypanosomes infestants dans les excréments une fois qu'ils ont été émis par l'Insecte. Théoriquement, on peut admettre qu'ils restent vivants aussi longtemps que les déjections sont humides et il faut se souvenir que dans les « ranchos » insalubres dans lesquels vivent les $R$. prolixus ce temps de dessication doit être long.

Plus significatives sont les expériences de Wood (1942) qui démontrent que les trypanosomes survivent pendant un mois dans les cadavres de Triatoma sp. tués par la chaleur ou morts naturellement. Cet auteur affirme la possibilité d'infestation des rongeurs par ingestion de réduves infestées. Quelques auteurs argentins de l'école de Mayer nous ont dit (1964) être parvenus à prouver la contagiosité des excréments même lorsqu'ils sont desséchés depuis peu de temps. Rappelons enfin que Juan C. Gomez Nuñez (1964) a réussi à infester des souris blanches en leur faisant dévorer des cadavres de $R$. prolixus vieux de trois jours et conservés à la température du laboratoire. Ces faits sont extrêmement importants à considérer au point de vue de la prophylaxie de la maladie de Chagas chez l'homme puisque après une pulvérisation d'insecticides dans l'habitation, les cadavres des $R$. prolixus infestés peuvent être dévorés par les chiens, les chats ou les rats, ce qui assurera malgré tout la diffusion du trypanosome.

Dans le cas de contamination buccale, le trypanosome doit traverser le tube digestif pour envahir l'organisme du chien. Quelle est alors l'importance de la barrière stomacale ? En théorie, le trypanosome devrait être attaqué et détruit par le suc gastrique. C'est ce point essentiel que nous avons tenté d'élucider expérimentalement. Il convient d'abord de citer Bordet, Coulon et Sevestre (1962), qui ont mesuré la durée du transit normal des aliments dans le tube digestif du chien. En utilisant plusieurs formules de repas, ces auteurs montrent que, radiologiquement, les temps de passage sont les suivants : œsophage, 5 à 20 secondes, pylore ouvert après une minute et jusqu'à 2 heures, fin de l'évacuation gastrique : 30 minutes à 4 heures, intestin vide en 5 à 12 heures, rectum vide au bout de 24 à 48 heures. On peut donc déduire de ces chiffres que le pylore est ouvert dès la première minute après l'ingestion et que, pour une durée aussi brève, les trypanosomes arrivent à traverser l'estomac sans dommage, surtout si le bol alimentaire contient du lait ou si c'est l'Insecte entier que le chien a ingéré.

A partir de ces notions, chez la plupart des animaux utilisés, nous avons réalisé l'infestation buccale en mélangeant les excréments positifs de $R$. prolixus à du lait. Dans tous les cas où le sang périphérique montrait des trypanosomes à l'examen à frais, nous avons prélevé quelques organes qui ont été adressés à l'Institut d'Anatomopathologie de la Faculté des Sciences vétérinaires de l'Université centrale du Venezuela. Les résultats de ces examens sont les suivants :

Intestin: Chez deux rats positifs sacrifiés 22 jours après l'infestation buccale, on trouve une discrète éosinophilie de la muqueuse. 
Foie: Chez un cobaye sacrifié 24 heures après la contamination existe une hépatite séreuse. Chez un autre, sacrifié au septième jour, existe une congestion discrète.

Chez une souris sacrifiée au $20^{\circ}$ jour, on trouve une hépatite interstitielle avec petits foyers de dégénérescence cellulaire et congestion. Chez deux rats sacrifiés positifs au $22^{\circ}$ jour, on observe une discrète congestion centrolobulaire avec hépatite interstitielle. Chez un troisième, dans des conditions identiques, existe une dégénérescence trouble de la cellule hépatique avec stase du premier degré. Ce même rat présente aussi un envahissement massif du cœur par des formes Leishmania ainsi que les cellules musculaires du poumon.

Un cobaye sacrifié positif au $22^{\circ}$ jour présente une dégénérescence graisseuse et une hépatite interstitielle. Chez un autre cobaye, dans les mêmes conditions, on trouve une hépatite interstitielle discrète. Chez un troisième existe une dégénérescence cellulaire en foyer et une congestion passive.

Chez un chien sacrifié positif au $20^{\circ}$ jour, existe une dégénérescence trouble et une dégénérescence vacuolaire en foyer. Ce chien a présenté une myocardite interstitielle aiguë sans Leishmania.

Chez un chien sacrifié positif au $36^{\circ}$ jour, on trouve une hépatite du premier degré avec dégénérescence trouble des cellules hépatiques. C'est ce chien qui avait des amas leishmaniens dans le cœur.

Chez un cobaye sacrifié positif au $22^{\circ}$ jour, existe une dégénérescence graisseuse, une hépatite interstitielle et des foyers d'infiltration d'origine probablement parasitaire. Deux cobayes sains du même élevage ont montré un foie sans lésion.

Cœur: Nous avons trouvé deux fois des Leishmania dans le cœur: la première chez un rat sacrifié au $22^{\circ}$ jour, avec invasion massive du cœur par les formes Leishmania; la seconde chez un chien sacrifié au $36^{\circ}$ jour.

Poumon: Chez le rat précédent, nous avons trouvé des Leishmania dans les fibres musculaires d'une artère de moyen calibre.

Ces examens histologiques nous permettent de supposer quelle est la voie suivie par les trypanosomes après la contamination buccale. Nous avons imaginé une nouvelle série d'expériences de façon à ce que les trypanosomes parviennent directement dans l'intestin.

Trois chiots de six semaines ont ingurgité des capsules de gélatine contenant des excréments positifs de $R$. prolixus dilués dans de l'eau physiologique. Ces capsules ont été introduites de force au fond de la gueule afin de ne pas être détériorées par les dents de l'animal. Nous avons trouvé des trypanosomes dans le sang périphérique 30 jours plus tard. Dans ce cas, la quantité de trypanosomes circulants était bien plus grande que celle que nous avions l'habitude d'observer. Ainsi, lorsque la contamination était faite par ingestion de lait virulent, nous ne trouvions qu'un trypanosome pour deux gouttes de sang alors que cette fois chaque goutte de sang contenait de nombreux trypanosomes. Pour connaître exactement le nombre de trypanosomes par millimètre cube de sang, nous avons utilisé l'hématimètre de Neubauer en diluant le 
sang au 1/20 avec de l'eau physiologique grâce à une pipette à numération de leucocytes. Le volume total de liquide était de $10 \mathrm{~mm}^{3}$ et nous avons trouvé :

- chez le chien $\mathrm{n}^{\circ}$ 1: 2 trypanosomes, soit 4 par $\mathrm{mm}^{3}$;

- chez le chien $\mathrm{n}^{\circ} 2: 25$ trypanosomes, soit 50 par $\mathrm{mm}^{3}$;

- chez le chien $\mathrm{n}^{\circ} 3: 8$ trypanosomes, soit 16 par $\mathrm{mm}^{3}$.

\section{Commentaires}

Les résultats positifs obtenus par l'administration des capsules de gélatine contenant $T$. cruzi démontrent la réalité du passage du parasite à travers la muqueuse intestinale. Si nous comparons ces résultats avec ceux obtenus par l'ingestion de lait contaminé, nous pouvons conclure que l'estomac représente bien un obstacle, qu'il assure la destruction d'une grande partie des trypanosomes ingérés mais qu'une certaine proportion d'entre eux parvient cependant à franchir cette barrière et à atteindre l'intestin.

L'existence d'une éosinophilie de la muqueuse intestinale chez deux rats contaminés par voie buccale permet d'affirmer qu'il s'agit de la trace du franchissement de la paroi par le trypanosome. Nous soulignons que ces rats n'hébergeaient aucun parasite intestinal.

La constance des lésions d'hépatite interstitielle chez les chiens, les cobayes et les rats nous permet de supposer qu'une fois traversée la paroi intestinale, les trypanosomes se localisent dans le foie où doit se constituer le foyer primaire. Rappelons que les cobayes témoins ne présentent pas de telles lésions hépatiques.

La découverte de formes Leishmania dans le cœur de deux chiens et d'un rat nous permet de maintenir la notion de la localisation définitive du trypanosome dans les fibres musculaires, en particulier celles du cœur.

\section{Conclusions}

1) L'ingestion des excréments de $R$. prolixus contaminé provoque, chez le chien, une maladie de Chagas.

2) Dans le tractus digestif, les trypanosomes sont capables de traverser la barrière chimique stomacale pour parvenir dans l'intestin.

3) Ils traversent ensuite la paroi intestinale pour passer dans le système porte.

4) Parvenus au foie, ils y entraînent la formation du foyer primaire, qui laisse des lésions d'hépatite interstitielle.

5) Après évolution de ce foyer primaire, les trypanosomes atteignent la circulation générale pour se rendre dans tout l'organisme et se localiser dans les fibres musculaires, en particulier celles du cœur. 


\section{III. - L' «épreuve du chipo» et son utilisation pour le diagnostic et l'expérimentation dans la maladie de Chagas}

Les expériences suivantes, faites avec $T$. cruzi et $T$. venezuelense, ont abouti à la mise au point d'une technique nouvelle ayant de multiples incidences théoriques et pratiques.

\section{Avec Trypanosoma venezuelense}

Nous pratiquons, chez le cobaye, l'injection intrapéritonéale de $0,5 \mathrm{~cm}^{3}$ du sang du çur du cobaye donneur. Les résultats sont irréguliers. Quelques animaux ont une parasitémie qui croît régulièrement jusqu'à la mort, d'autres meurent prématurément pour une cause non déterminée, d'autres enfin présentent une très faible positivité du sang périphérique, qui alterne avec des phases négatives pour lesquelles il est impossible d'invoquer des crises trypanolytiques.

\section{$1^{\circ}$ Epreuve du * chipo pour le diagnostic.}

Chez un cobaye qui s'est montré très faiblement positif (un à deux trypanosomes par frottis du sang de l'oreille) on examine, le 5 avril, deux gouttes de sang qui ne contiennent aucun trypanosome. Sur cet animal apparemment négatif, on fait gorger trois nymphes de $R$. prolixus de notre élevage. Les Insectes sont sacrifiés immédiatement, une heure et 24 heures après le repas. Chez les deux premiers, lé sang contenu dans l'estomac est fortement positif alors qu'il est négatif pour le troisième.

A partir de cette expérience, nous avons systématiquement pratiqué cette « épreuve du chipo »lorsque le sang périphérique des animaux inoculés se révélait négatif. A plusieurs reprises, nous avons constaté que cette épreuve était positive avant que ne le soit l'examen de sang classique et que, de toute façon, lorsque le sang était positif, on trouvait beaucoup plus de trypanosomes dans le sang ingéré par le vecteur. A l'inverse, nous n'avons jamais obtenu une « épreuve du chipo » négative lorsque le sang périphérique était positif.

Chez la souris infectée par $T$. venezuelense, la phase d'incubation est de 24 à 72 heures. Pour tenter d'obtenir un allongement de cette durée, nous avons inoculé à un lot de huit souris le sang contenu dans l'estomac d'un e chipo » gorgé sur un cobaye positif. Ce sang a été dilué dans de l'eau physiologique stérile jusqu’à ce que la concentration des trypanosomes devienne inférieure à un trypanosome par champ au grossissement de 400 diamètres. On injecte en sous-cutané $0,05 \mathrm{~cm}^{3}$ du mélange.

L'examen quotidien du sang des animaux donne les résultats résumés dans le tableau suivant: 
TABleau II

\begin{tabular}{|c|c|c|c|c|c|c|c|c|}
\hline & $10 \mathrm{j}$. & 11 & 12 & 13 & 14 & 15 & 16 & 17 \\
\hline Souris $56 \ldots \ldots \ldots$ & $\mathrm{Ch}$ & $\mathrm{s}$ & & & & & & \\
\hline Souris $58 \ldots \ldots \ldots$ & & & $\mathrm{Ch}$ & & $\mathrm{s}$ & & & \\
\hline Souris $59 \ldots \ldots$ & Ch & $\mathrm{s}$ & & & & & & \\
\hline Souris $63 \quad \ldots \ldots \ldots$ & $\mathrm{Ch}$ & & $\mathrm{Ch}$ & & $\mathrm{Cb}$ & & & $\mathrm{s}$ \\
\hline Souris $66 \ldots \ldots \ldots$ & & & & $\mathrm{Ch}$ & $\mathrm{s}$ & & & \\
\hline Souris $67 \ldots \ldots \ldots$ & & & $\mathrm{Ch}$ & & $\mathrm{S}$ & & & \\
\hline Souris $71 \ldots \ldots \ldots$ & & $\mathrm{Ch}$ & $\mathrm{Ch}$ & & $\mathrm{Ch}$ & & $\mathrm{s}$ & \\
\hline Souris $73 \ldots$ & & $\mathrm{Ch}$ & $\mathrm{s}$ & & & & & \\
\hline
\end{tabular}

$\mathrm{S}=+$ sang. $\mathrm{Ch}=+\ll$ chipo .

\section{$2^{\circ}$ Epreuve du «chipo» pour l'inoculation.}

Sur un cobaye fortement positif, on fait piquer plusieurs Insectes. Le sang contenu dans l'estomac montre de nombreux trypanosomes. On injecte $1 \mathrm{~cm}^{3}$ intra-péritonéale à un chien et $0,5 \mathrm{~cm}^{3}$ en intra-péritonéale à trois cobayes. Tous les animaux sont positifs le $15^{\circ}$ jour, les cobayes meurent le $20^{\circ}$ jour et le chien le $30^{\circ}$.

Une souris infestée sert de donneur. On inocule le sang contenu dans l'estomac des Insectes à la dose de $0,2 \mathrm{~cm}^{3}$ en intra-péritonéale à deux cobayes qui sont positifs un mois plus tard et morts au $42^{\circ}$ jour.

Des résultats identiques ont été obtenus de façon constante dans toute une série d'expériences similaires. Ceci nous conduit à reproduire cette expérimentation avec $T$. cruzi pour lequel l'utilisation de $R$. prolixus est, en théorie, particulièrement indiquée.

\section{Avec Trypanosoma cruzi}

Lot $n^{\circ}$ 1: Quatre souris inoculées en intra-péritonéale avec $0,2 \mathrm{~cm}^{3}$ de culture. Les résultats obtenus sont résumés dans le tableau III.

Il faut remarquer que dans les cas où l' " épreuve du chipo » et l'examen du sang périphérique ont été positifs au même moment, l' « épreuve » est toujours plus fortement positive que le sang.

Lot $n^{\circ} 2$ : Trois souris inoculées avec le sang gastrique d'un $R$. prolixus immédiatement après qu'il se soit gorgé sur la souris $\mathrm{n}^{\circ} 3$ du lot précédent au moment où cet animal était fortement positif. Les résultats obtenus sont résumés dans le tableau IV. 
TABLEAU III

\begin{tabular}{|c|c|c|c|c|c|c|c|c|c|c|c|c|}
\hline & $\dot{\tilde{N}}$ & 25 & 30 & 33 & 34 & 48 & 54 & 56 & 68 & 72 & 78 & 134 \\
\hline $\begin{array}{c}\text { Sang } \ldots . \\
\text { Souris } 1 \\
\text { Chipo } \ldots\end{array}$ & - & + & - & $\begin{array}{l}+ \\
+\end{array}$ & $\begin{array}{l}+ \\
+\end{array}$ & ++ & + & $\begin{array}{l}- \\
+\end{array}$ & $\begin{array}{l}- \\
++\end{array}$ & $\begin{array}{l}- \\
+\end{array}$ & $\begin{array}{l}- \\
-\end{array}$ & - \\
\hline $\begin{array}{r}\text { Sang } \ldots . \\
\text { Souris } 2 \\
\text { Chipo } \ldots\end{array}$ & - & - & + & ++ & ++ & $++t+$ & + & + & $\begin{array}{l}- \\
++\end{array}$ & $\begin{array}{l}- \\
+\end{array}$ & $\begin{array}{l}- \\
+\end{array}$ & - \\
\hline $\begin{array}{l}\text { Sang } \ldots . \\
\text { Souris } 3 \\
\text { Chipo } \ldots\end{array}$ & - & - & +++ & ++ & + & $\begin{array}{l}- \\
++\end{array}$ & $\begin{array}{l}- \\
+\end{array}$ & $\begin{array}{l}+ \\
+\end{array}$ & + & $\begin{array}{l}- \\
+\end{array}$ & $\begin{array}{l}- \\
+\end{array}$ & \begin{tabular}{l||}
- \\
+
\end{tabular} \\
\hline $\begin{array}{l}\text { Sang } \ldots . \\
\text { Souris } 4 \\
\text { Chipo } \ldots\end{array}$ & - & - & ++ & ++ & ++ & ++ & + & + & - & $\begin{array}{l}- \\
+\end{array}$ & $\begin{array}{l}- \\
+\end{array}$ & - \\
\hline
\end{tabular}

TABLEAU IV

\begin{tabular}{|c|c|c|c|c|c|c|c|c|c|}
\hline & $18 \mathrm{j}$. & 19 & 20 & 38 & 46 & 52 & 61 & 68 & 72 \\
\hline $\begin{array}{l}\text { Sang } \ldots \ldots \\
\text { Souris } 6 \\
\text { Chipo } \quad \ldots .\end{array}$ & ++ & + & $\begin{array}{l}++ \\
++\end{array}$ & + & + & $\begin{array}{l}+ \\
+\end{array}$ & & & \\
\hline $\begin{array}{r}\text { Sang } \ldots \ldots \\
\text { Souris } 7 \\
\text { Chipo } \ldots .\end{array}$ & - & $\begin{array}{l}- \\
++\end{array}$ & $\begin{array}{r}+ \\
++\end{array}$ & + & $\begin{array}{l}- \\
+\end{array}$ & + & - & - & $\begin{array}{l}- \\
+\end{array}$ \\
\hline $\begin{array}{r}\text { Sang } \ldots . \cdots \\
\text { Souris } 8 \\
\text { Chipo } \quad \ldots \ldots\end{array}$ & - & $\begin{array}{l}- \\
+\end{array}$ & $\begin{array}{r}+ \\
++\end{array}$ & $\begin{array}{r}+ \\
++\end{array}$ & $\begin{array}{l}- \\
+\end{array}$ & + & - & $\begin{array}{l}- \\
+\end{array}$ & - \\
\hline
\end{tabular}

Au $72^{\circ}$ jour, pour connaître la durée pendant laquelle l' « épreuve du chipo » pouvait être utilisable, nous faisons gorger quatre nymphes de $R$. prolixus sur la souris $\mathrm{n}^{\circ}$ 7. Le sang' contenu dans l'estomac des Insectes disséqués respectivement immédiatement, une heure et cinq heures après le repas, est fortement positif. Chez le quatrième, disséqué à la sixième heure, le sang gastrique est négatif.

Lot $n^{\circ} 3$ : Quatre souris inoculées en intra-péritonéale avec $0,2 \mathrm{~cm}^{3}$ de culture de notre souche de $T$. cruzi. Les résultats obtenus sont résumés dans le tableau $\mathrm{V}$.

Annales de Parasitologie humaine et comparée (Paris), t. 41, 1966, $\mathrm{n}^{\circ} 6$ 
Tableau $\mathrm{V}$

\begin{tabular}{|c|c|c|c|c|c|c|}
\hline & $11 \mathrm{j}$. & 25 & 32 & 35 & 36 & 37 \\
\hline $\begin{array}{r}\text { Sang } \\
\text { Souris } 9 \\
\text { Chipo } \\
\end{array}$ & $\begin{array}{l}+ \\
+\end{array}$ & $\begin{array}{l}- \\
+\end{array}$ & - & $\begin{array}{l}- \\
+\end{array}$ & $\begin{array}{l}+ \\
-\end{array}$ & + \\
\hline $\begin{array}{l}\text { Sang } \ldots \ldots \ldots \\
\text { Souris } 10 \\
\text { Chipo } \ldots \ldots\end{array}$ & $\begin{array}{l}- \\
++\end{array}$ & $\begin{array}{l}- \\
+\end{array}$ & $\begin{array}{l}- \\
-\end{array}$ & - & - & + \\
\hline $\begin{array}{l}\text { Sang } \ldots \ldots \ldots \\
\text { Souris } 11 \\
\text { Chipo } \\
\end{array}$ & - & - & - & - & - & - \\
\hline $\begin{array}{l}\text { Sang } \ldots . . . . \\
\text { Souris } 12 \\
\text { Chipo } \\
\ldots \ldots . .\end{array}$ & - & - & $\begin{array}{l}- \\
+\end{array}$ & - & $\begin{array}{l}- \\
+\end{array}$ & + \\
\hline
\end{tabular}

Il convient de retenir particulièrement le cas de la souris $\mathrm{n}^{\circ} 11$ dont le sang a toujours été négatif mais chez qui l' "épreuve de chipo » a été une seule fois positive.

Lot $n^{\circ} 4$ : Ces deux chiens ont également servi pour une expérience que nous exposerons ultérieurement. Le chien a ingéré les trypanosomes le 31 mai 1965. Les résultats obtenus sont résumés dans le tableau VI.

TABLEAU VI

\begin{tabular}{|c|c|c|c|c|}
\hline & $31-5-65$ & $16-6-65$ & $23-6-65$ & $28-6-65$ \\
\hline Chien 1 . & Contamination & $s-$ & $s-$ & $\underset{\mathrm{Ch} .+}{\mathrm{S}-}$ \\
\hline Chien 2 . & Contamination & $s-$ & $s-$ & $\underset{\mathrm{Ch} .+}{\mathrm{S}-}$ \\
\hline Chien 3 . & Témoin & $s-$ & $s-$ & $\stackrel{\mathrm{S}-}{\mathrm{Ch}-}$ \\
\hline Chien 4 . & Témoin & $s-$ & $s-$ & $\frac{\mathrm{S}-}{\mathrm{Ch}-}$ \\
\hline
\end{tabular}




\section{Conclusions}

De ces expériences, on peut conclure que l' « épreuve du chipo » est une méthode de diagnostic plus sûr que l'examen du sang périphérique des animaux inoculés tant avec $T$. cruzi qu'avec $T$. venezuelense. A plusieurs reprises, la positivité de l' "épreuve du chipo » a été plus précoce que l'examen du sang. De plus, cette expérimentation nous prouve que le sang contenu dans l'estomac des réduves reste positif jusqu'à cinq heures après le repas. Enfin, la raison pour laquelle on trouve plus de trypanosomes dans le sang ingéré que dans le sang périphérique est peut-être que le $R$. prolixus introduit ses pièces piqueuses dans l'intimité des tissus et aspire ainsi le sang capillaire ou même le sang lacunaire. Ceci pourrait indiquer que les trypanosomes, absents du sang périphérique, se réfugient dans le réseau capillaire et dans le sang lacunaire.

L'utilisation de l' « épreuve du chipo » pour prélever le sang des animaux positifs en vue de la réinoculation constitue une excellente méthode, sans danger pour le donneur tant avec $T$. cruzi qu'avec $T$. venezuelense. On peut ainsi conserver vivants les donneurs et continuer à observer le déroulement de leur maladie.

\section{$* *$ \\ IV. - Rôle de la Mouche domestique dans la transmission de la maladie de Chagas}

\section{Travaux antérieurs}

Ce sont les travaux de Vergani et de Mayer qui nous ont donné l'idée de cette expérimentation et nous allons en exposer ici l'essentiel, car il nous paraît qu'ils n'ont pas eu la diffusion qu'ils méritaient. En 1912, à Panama, Darling montre l'intervention de Musca domestica dans la transmission des trypanosomiases en prouvant qu'elle peut véhiculer $T$. hippicum dans sa trompe.

En 1934, Thomson et Lamborn étudient le transport de T. brucei par Musca spectanda et montrent la contagiosité des excréments de la mouche lorsqu'elle se gorge de sang contenant le trypanosome.

En 1952, Vergani, au Venézuela, reprend ces travaux avec $T$. venezuelense et $T$. cruzi d'une part et les « vecteurs $M$. domestica et Callitrota macellaria. Ce travail contient bien des expériences de grande valeur et nous allons en extraire ce qui a trait à $M$. domestica et $T$. cruzi. Vergani a aussi étudié la transmission par l'appareil buccal de la mouche, mais nous ne parlerons que des expériences faítes avec les déjections de l'Insecte. A propos des inoculations d'excréments de mouches gorgées du sang de cobaye porteur de $T$. cruzi, Vergani écrit :

«Dans ce cas, l'examen microscopique des excréments et du contenu intestinal des Insectes disséqués ne nous permettent pas des conclusions définitives. En effet, les 
trypanosomes sont très peu nombreux et nous n'avons que fort peu de chances de les voir dans le champ du microscope. Pour connaître la longévité de Schizotrypanum cruzi dans l'intestin de $M$. domestica nous avons inoculé à des souris le contenu intestinal d'Insectes disséqués et obtenu des résultats positifs jusqu’à la huitième heure après le repas de sang.

«Nous avons immédiatement inoculé les déjections d'Insectes émis à divers intervalles de temps après leur repas infestant. Nous avons fait les expériences suivantes:

« Premier essai : Nous récoltons 16 échantillons de déjections de cinq minutes à une heure après le repas des Insectes et les inoculons séparément à 16 souris dont 12 se montrent positives.

« Second essai : Nous récoltons 18 échantillons d'une à deux heures après le repas et les inoculons séparément à un nombre égal de souris dont 10 se montrent positives.

«Troisième essai : Nous récoltons 22 échantillons de deux à trois heures après le repas. Sur les 22 souris inoculées, 4 ont été positives. La dernière souris positive est celle qui fut inoculée avec les déjections recueillies 2 heures 45 après l'absorption de sang. »

Il est également intéressant de transcrire la dernière partie du texte de Vergani qui se révèle prophétique :

« C'est un fait prouvé que les trypanosomes peuvent pénétrer dans l'organisme animal à travers les muqueuses ou la peau humide ainsi que l'affirme Emile Brumpt. Nous avons l'intention de faire une étude comparative des possibilités de pénétration à travers les différentes muqueuses de $T$. venezuelense et $T$. cruzi. Parce que les Diptères non vulnérants se trouvent constamment autour des muqueuses des Mammifères et de l'homme, il est important d'établir si ces Insectes sont capables de prélever des trypanosomes au niveau d'une solution de continuité de la peau et de les déposer avec leurs excréments sur les muqueuses, transmettant ainsi une trypanosomiase. Nous pouvons déjà faire état d'un premier résultat positif puisqu'avec les excréments de $M$. domestica contenant des $T$. cruzi nous avons, en les ayant déposés sur la muqueuse oculaire de 47 souris, obtenu une inoculation positive. Cet unique résultat ne nous permet pas de conclure pour le moment. »

Nous devons, en second lieu, citer le travail de Mayer (1961), dont nous avons fait mention dans la première partie de ce travail. Rappelons que Mayer a démontré la transmission par voie buccale que nous avons pleinement confirmée. Dans son article, Mayer expose sa théorie sur le mécanisme de la transmission de la maladie de Chagas dans les termes suivants :

« Nous avons montré que le lait peut servir de véhicule aux trypanosomes métacycliques. La contamination par les déjections des Triatomides est fréquente car c'est un fait bien connu que ces Insectes laissent tomber leurs excréments du haut des toits de paille qui leur servent de refuge. Les biberons, les verres, les tables et les autres ustensiles de cuisine peuvent être contaminés et devenir ainsi une source importante d'infestation par voie digestive... »

\& La piqûre de l'Insecte est peu douloureuse et provoque un prurit local. C'est un fait souligné par d'autres chercheurs que l'homme peut se contaminer lui-même si, après s'être gratté, il porte ses doigts souillés au niveau de la muqueuse buccale... » « Nous pouvons dire 
que, chez le chien et le chat, la porte d'entrée la plus importante est la voie digestive, soit par ingestion d'aliments contaminés, ou par léchage et déglutition des déjections des Insectes déposées sur leur corps. 》

\section{Hypothèses de travail}

A la lecture de ces travaux et à la lumière de nos propres expériences sur la contamination par voie buccale qui donne $100 \%$ de résultats positifs, il nous est apparu évident qu'il fallait réviser les notions classiquement admises sur le mode de transmission de la maladie de Chagas chez les Mammifères et, plus spécialement, chez l'homme. Pour nous, il nous paraît difficilement admissible de continuer à affirmer avec autant de force que le font les auteurs les plus illustres que l'homme se contamine en portant à ses yeux ses doigts souillés des excréments de $R$. prolixus. Nous pensions que ce mécanisme de transmission, tout comme dans le cas des souris de Vergani infestées par voie oculaire, était peut-être essentiellement lié aux excréments de $M$. domestica. De plus, si cette mouche est capable de véhiculer les trypanosomes et d'héberger des formes pathogènes dans ses excréments, il existe certainement des probabilités plus grandes pour qu'en règle, l'homme et les Mammifères se contaminent par ingestion de ces déjections lorsque l'Insecte les dépose sur leur peau ou qu'il les laisse tomber sur les aliments, en particulier le lait. Cette hypothèse fut exposée par nous, en septembre 1964, au Congrès mondial de Parasitologie, à Rome, mais à cette époque nous ne pouvions insister sur ce point, faute de preuves expérimentales.

L'affirmation de Mayer selon laquelle les excréments de $R$. prolixus tombent du haut des toits sur les objets et les aliments disposés dans le « rancho » ne nous a pas totalement convaincu. Nous devons garder présents à l'esprit deux faits importants pour la transmission de la maladie chez le chien et chez l'homme :

$1^{\circ}$ les excréments de $M$. domestica sont pratiquement invisibles ;

$2^{\circ}$ c'est un fait d'observation courante que les chiens dévorent les mouches qui les importunent alors qu'il est bien plus rare qu'ils aient l'occasion de manger des \& chipos $\gg$.

Pour tenter de démontrer cette hypothèse de travail, nous avons réalisé les expériences suivantes.

\section{Expériences réalisées}

Sur une lame de Willis, nous avons récolté la totalité du contenu intestinal de $\operatorname{deux} R$. prolixus positifs à $T$. cruzi par pression sur les abdomens. Nous utilisons un lot de $10 \mathrm{M}$. domestica capturées depuis quelques jours. Les mouches sont placées dans un verre renversé au-dessus des excréments de $R$. prolixus. Nous observons que les Insectes absorbent cet « appât \$ avec avidité. Après 20 minutes d'emprisonnement, nous retirons les mouches qui sont, 10 minutes plus tard, broyées avec des ciseaux. Deux lots de 5 mouches broyées sont mélangés avec du lait et chacun est proposé à 
un chien de deux mois qui l'absorbe sans difficulté. Le lendemain, nous répétons cette « inoculation » avec 26 mouches alimentées pendant 45 minutes. Après broyage et mélange avec du lait, chaque chien reçoit 13 mouches. Le troisième jour, nouvelle « inoculation avec 7 mouches pour chaque chien. Au total, ces chiots de deux mois ont chacun absorbé $25 \mathrm{M}$. domestica nourries sur les déjections positives de $R$. prolixus. Deux chiots du même élevage sont gardés comme témoins. Le tableau $\mathrm{n}^{\circ}$ VII résume les résultats obtenus.

TABLEAU VII

\begin{tabular}{|c|c|c|c|c|c|c|}
\hline & $1^{\text {er }}$ jour & $2^{\circ}$ jour & $3^{\circ}$ jour & $16 \mathrm{j}$. & $23^{\circ} \mathrm{j}$. & $26^{\circ}$ jour \\
\hline Chien 1. & 5 mouches & 13 mouches & 7 mouches & $s-$ & $s-$ & $\mathrm{S}-$ \\
\hline Chien 2 . & 5 mouches & 13 mouches & 7 mouches & $\mathrm{s}-$ & $s-$ & $\frac{\mathrm{S}-}{\mathrm{Ch}+}$ \\
\hline Chien 3 . & témoin & témoin & témoin & $s-$ & $\mathrm{S}-$ & $\frac{\mathrm{S}-}{\mathrm{Ch}-}$ \\
\hline Chien 4 . & témoin & témoin & témoin & $s-$ & $s-$ & $\frac{\mathrm{S}-}{\mathrm{Ch}-}$ \\
\hline
\end{tabular}

$\mathrm{S}=$ sang. $\mathrm{Ch}=$ épreuve du $«$ chipo $»$.

TABLEAU VIII

\begin{tabular}{|c|c|c|c|c|c|c|c|}
\hline & $1^{\text {er }}$ jour & $2^{\circ}$ jour & $24^{\circ} \mathrm{j}$ & $25^{\circ} \mathrm{j}$. & $28^{\circ} \mathrm{j}$. & $30^{\circ} \mathrm{j}$ & $35^{\circ} \mathrm{j}$. \\
\hline Chien $25 \ldots$ & 10 mouches & 10 mouches & $\frac{\mathrm{s}-}{\mathrm{Ch}-}$ & $\frac{\mathrm{s}-}{\mathrm{Ch}+}$ & $\frac{\mathrm{S}-}{\mathrm{Ch}+}$ & $\stackrel{\mathrm{S}-}{\mathrm{Ch}+}$ & \\
\hline Chien $76 \ldots$ & 10 mouches & 10 mouches & $\stackrel{\mathrm{S}-}{\mathrm{Ch}-}$ & $\stackrel{\mathrm{S}-}{\mathrm{Ch}-}$ & $\stackrel{\mathrm{S}-}{\mathrm{Ch}-}$ & $\stackrel{\mathrm{S}-}{\mathrm{Ch}-}$ & $\mathrm{S}-$ \\
\hline Chien $78 \ldots$ & 10 mouches & 10 mouches & $\mathrm{S}-$ & $\mathrm{S}-$ & $\stackrel{\mathrm{S}-}{\mathrm{Ch}-}$ & $\stackrel{\mathrm{S}-}{\mathrm{Ch}-}$ & $\mathrm{Ch}-$ \\
\hline
\end{tabular}

$\mathrm{S}=$ sang. $\mathrm{Ch}=$ épreuve du $\ll$ chipo $»$.

Une seconde série d'expériences plus rigoureuses est entreprise pour savoir si les mouches hébergeaient bien le trypanosome dans leur tube digestif et si elles pouvaient assurer la transmission pendant un certain laps de temps. Un lot de $M$. domestica 
capturées dans notre propre demeure est nourri sur les excréments de $R$. prolixus positifs à $T$. cruzi. Les mouches se nourrissent pendant 45 minutes puis sont tuées par immersion dans l'eau. Elles sont ensuite séchées dans une boîte de Pétri placée sous le rayonnement d'une lampe de 60 watts pendant une heure. Nous éliminons ensuite la tête, le thorax, les pattes et les ailes, ne conservant que les abdomens. Les chiots $\mathrm{n}^{\circ s} 25,76$ et 78 reçoivent chacun 10 abdomens de mouches mélangés à du lait. La même « inoculation » est faite le lendemain (10 abdomens à chaque chien). Au total, ces chiots de deux mois ont reçu 20 abdomens de $M$. domestica pendant deux jours consécutifs. L'examen à frais du sang périphérique et l' « épreuve du chipo * donnent les résultats résumés dans le tableau $\mathrm{n}^{\circ}$ VIII.

\section{Conclusions}

Ces expériences prouvent qu'il est possible de contaminer expérimentalement des chiens par $T$. cruzi en leur faisant ingérer du lait contenant des $M$. domestica nourries avec des excréments de $R$. prolixus positifs. La première expérience a donné $100 \%$ de réussite et la seconde deux résultats positifs sur trois. Dans ce second cas, l'expérience a été faite dans des conditions plus strictes. Nous croyons cependant que si les résultats ont été moins fortement positifs que dans la première expérience, c'est parce que les chiots n'ont mangé des mouches infestées que deux jours consécutifs au lieu de trois. Il est certain que dans les conditions naturelles le chien mange des mouches à plusieurs reprises. Il faut en outre souligner que les deux chiens positifs $\left(\mathrm{n}^{\circ \mathrm{s}} 25\right.$ et 76) ne l'ont été qu'à l' « épreuve du chipo », alors que, jusqu'au moment où nous rédigeons ce travail (trente-six jours plus tard), le sang périphérique est négatif à l'examen à frais. Ceci prouve que l' « épreuve du chipo » est indispensable pour ce genre de travail. Très certainement, dans nos expériences comme dans celles des autres auteurs, certains animaux considérés comme négatifs parce que l'on n'a eu recours qu'à l'examen direct du sang périphérique se seraient montrés positifs si l'on avait utilisé l' « épreuve du chipo ».

Il est logique de penser que, dans les * ranchos chagasiques , ce sont les mouches ( $M$. domestica) qui, en se nourrissant des déjections de $R$. prolixus, habitants des toits et des murs, prélèvent les $T$. cruzi et assurent leur dispersion sur les aliments (lait en particulier) et les divers objets de l'habitation. Leurs excréments pratiquement invisibles contiennent des trypanosomes infestants. Il se peut également que l'infestation se fasse par ingestion de la mouche elle-même.

\section{V. - Une nouvelle théorie sur la transmission de la maladie de Chagas}

Nous pouvons penser qu'à partir des réservoirs de virus sauvages (Tatous, etc.), le $T$. cruzi parvient à s'établir dans les palmeraies et les fourrés de cactées où vivent les $R$. prolixus qui vont l'héberger. Ces réduves pénètrent dans l'habitation humaine, 
soit du fait de l'homme lui-même lorsqu'il utilise les palmes pour construire le toit de son « rancho » ou les cactus pour en bâtir les murs, soit, dans les habitations en dur, en volant la nuit ou amenés avec le bois qui sert de combustible. Quoi qu'il en soit, ils s'établissent dans les murs et le toit du « rancho » où ils forment de grands rassemblements. La nuit, ils sortent de leurs refuges pour se nourrir sur les occupants du « rancho ( (homme, chien, chat, rongeurs, etc.).

Une fois dans la place, les $R$. prolixus sont infestés par $T$. cruzi en se gorgeant sur des hommes ou des animaux réservoirs de virus, en particulier les chiens de chasse. Les \& chipos " infestés maintiennent le cycle biologique du trypanosome en se nourrissant sur l'homme et les Mammifères. Il est indéniable que, tout au long de ce processus, l'infestation par voie oculaire ou cutanée est possible. Pour nous, ces voies ne sont pas les plus probables ainsi que le prouve, pour le Venezuela, la statistique de Pifano: syndrome ophtalmoganglionnaire $22 \%$, et chagome $6,66 \%$. En accord avec nos résultats expérimentaux, le mode habituel, surtout chez le chien, passe par $M$. domestica nourrie des excréments frais de $R$. prolixus infestés par $T$. cruzi et déposés dans les fentes des murs ou des toits. La mouche assure la dissémination de ces excréments dans le « rancho ». Ses propres déjections contiennent $T$. cruzi qui, de cette façon, vient contaminer les objets disposés dans l'habitation (couverts, outils, vêtements, etc.). Grâce à ces diverses sources, le chien, l'homme et les autres Mammifères peuvent s'infester par voie buccale. Dans le cas du chien, ce mécanisme est favorisé par la fréquence avec laquelle le chien dévore les mouches qui l'importunent.

Lorsque l'homme et les Mammifères ont ingéré les excréments de mouche infestés par T. cruzi (ou la mouche entière dans le cas du chien), le trypanosome traverse l'estomac, parvient dans l'intestin et passe dans le foie où se produit la lésion primaire qui précède la dissémination dans tout l'organisme. Le témoin de cette lésion initiale est une hépatite centrolobulaire qui, pour nous, serait spécifique puisque nous l'avons observée chez tous les animaux ayant ingéré des trypanosomes et qu'elle manque chez les témoins. A partir de ce foyer hépatique, le trypanosome envahit tout le corps en se localisant dans la musculature, en particulier celle du cœur. En ce qui concerne les formes sanguines, nous pensons qu'elles sont plus abondantes dans le réseau capillaire et dans le système lacunaire d'où nous l'avons extrait grâce à l' * épreuve du chipo ».

Il est certain que dans les rares cas où se produit la contamination oculaire ( $22 \%$ des cas au Venezuela), elle peut se faire plus fréquemment par les excréments de la mouche que par ceux des « chipos \$.

\section{Summary}

In the first part, Trypanosoma cruzi is transmitted to dogs, rats, white mice and guinea pigs by giving faeces of Rhodnius prolixus mixed with milk, to the animals. In the second part, the experience is repeated but faeces of Rhodnius prolixus are administred in gelatin capsules. The results are 100 per cent positive, and the conclusions are as follows : 
1. The dog is able to acquire the Chagas disease by ingestion of contaminated faeces from Rhodnius prolixus; 2 . Trypanosomes are able to follow the digestive tract and pass through the stomach arriving in the intestine; 3 . The trypanosomes pass through the intestinal wall and from the liver are distributed through the animal localising in the muscles, especially in the heart.

In the third part, the test for the Rhodnius prolixus is described. This test consists in feeding the Rhodnius prolixus on animals infected with Trypanosomes, and using the Insect's blood immediately for diagnosis and for inoculation.

In its diagnostic use, the test for the Rhodnius prolixus proves to be more precocious and more positive than the examination of fresh-blood of animals inoculated for experimental purposes with Trypanosoma venezuelense and with Trypanosoma cruzi. The author demonstrates the test is effective during at least five hours, using only one Insect for each test.

In inoculations, it has proved to be extremely useful, as the inoculations made with material extracted from the stomach of Rhodnius prolixus which had fed on animals with $T$. venezuelense and $T$. cruzi turned out to be 100 per cent positive.

The author had flies fed on the faeces of Rhodnius prolixus with Trypanosoma cruzi and then gave these flies mixed with milk to two-month-old-pippies. Each dog eats a total of 25 flies (Musca domestica) in three consecutive days. After 28 days, the dogs were negative in peripheric blood, but the test for the Rhodnius prolixus was carried out, it was highly positive. The negative tests made on the other two dogs in the same lot were used as a control.

A second lot made up of 3 two-month-old dogs was given milk containing the abdomens of flies which had fed on faeces of Rhodnius prolixus ( + for $T$. cruzi) and which had been dried for one hour under a 60 watt lamp. Each dog consumed the abdomens of 10 flies the first day, and of 10 others on the following day. On the 25 th day, one of the dogs was positive for the Rhodnius prolixus test. On the 35th day, the second was positive to the test, whereas the third dog it still under observation.

Finally, in conclusion, the author presents a new theory regarding the transmission of Chagas disease in Vertebrates, considering that, in addition to its natural source (the armadillo, etc.) the Trypanosoma cruzi succeeds in lodging in plants, mainly in the palms and cardoons where Rhodnius prolixus with trypanosomes are already found. The infected Rhodnius prolixus can be transferred to human dwellings by man himself when he cuts the leaves of the palm trees or uses parts of the cardoon also found in well constructed homes, due to the plants found in the surrounding area, either because the Insect has flown in at night, or has been taken in with firewood. In any case, the Rhodnius prolixus are found preferably in the roofs and halls of the « ranchos » where they form large colonies and emerge at night to feed on the inhabitants of the " rancho », human beings as well as animals (dogs, cats, rodents, etc.).

When once the Rhodnius prolixus have established themselves in the dwelling, they are infected by Trypanosoma cruzi after feeding on animals or human beings 
who are carriers of the trypanosome. It is interesting to indicate the important part which hunting dogs can play.

The infected Rhodnius prolixus maintain their biological cycle by feeding at night on men and animals, and during this process, there is no doubt that infections can be obtained by the ocular or mucous paths, cutaneously, etc., although it does not seem very probable as is shown in Venezuela by the findings of Pifano (1962) where the ophthalmoganglionar syndrome occurs in only 22 per cent of the patients and cutaneous chagoma in only 6,66 per cent.

In accordance with our experience, particularly in dogs, it is most probable that Musca domestica when feeding in the crevices of the walls and of the roof on the faeces of Rhodnius prolixus infected by $T$. cruzi spreads its faeces throughout the dwelling. These faeces of the flies contain the $T$. cruzi, which in this way gets into various foods (milk, cheese, etc.), or simply soils different objects in the dwelling (table, wares, tools, clothes, etc.) and by means of these objects, the trypanosomes can infect dogs, man and other Vertebrates orally. In the case of the dog, the mechanism would be favorable due to the frequency with which dogs eat the flies which bother them insistently.

After man or animals have ingested faeces of flies positively charged with $T$. cruzi, or after the dogs have ingested infected flies, the trypanosomes pass through the stomach, reach the intestine, pass through the intestinal walls, and by the portal system, reach the liver where they produce the first lesion before they are spread through the organism. The mark left is a centrolobular hepatitis, which can be considered as a specific sign observed in all the animals which had been fed with trypanosomes, but which has not been evident in the control. From the hepatic focus, the trypanosome spreads throughout the body establishing itself in the muscles, especially in the heart; and as far as the hematic forms are concerned, it seems that they are found more abundantly in the capillary blood and the lacunar system where we have obtained them by means of the « Test for Rhodnius prolixus 》.

There is no doubt that in the exceptional cases in which the contamination is ocular ( 22 per cent of the cases in Venezuela), it is more probably due to the faeces of flies than to the faeces of Rhodnius prolixus.

\section{Bibliographie}

Bordet, Coulon (J.) et Sevestre (J.), 1962. - Le repas d'épreuve en radiologie. Méthodologie d'analyse radiographique du transit digestif des Carnivores domestiques. Rec. Med. Vet., CXXXVIII, 4, 249-256.

DARLING (S. T.), 1962. - Experimental infection of the mule with Trypanosoma hippicum by means of Musca domestica. J. Exper. Med., 15 (4), 365-366.

-, 1912. - Notes on transmission of Tr. hippicum by means of Musca domestica. Proc. Canal zone Med. Ass. Mount Hope., Oct. 1911-Mar. 1912, 4 (2), 82.

-, 1913. - The part played by flies and other insects in the spread of infectious diseases in the tropics with special reference. Hippicum by Musca domestica Tr. 15. Internat. Cong. Hyg. and Demog., Wash., Sept. 23-28 1912, 4 (1), 182-185. 
Diaz-Ungria (C.), 1964. - Transmisión experimental del Trypanosoma cruzi en los vertebrados, I. - Contaminación bucal a partir de heces de Rhodnius prolixus infectados. Rev. Vet. Venezolana, XVI, 95, 341-352, Junio de 1964.

Diaz-Ungria (C.), 1964. - Transmisión experimental del Trypanosoma cruzi en los vertebrados. II. Camino que sigue el Trypanosoma en el organismo de los vertebradas cuando se les contamina por la via bucal; Rev. Vet. Venezuelana, XVII, 96, 3-13.

Dominguez Quesada (J.), 1964. - Investigaciones sobre la enfermedad de Chagas en el municipio José María Blanco, del Estado Lara. Boletín informativo de la Dir.de Malariología y Saneamiento Ambiental, IV, 1, 41-51.

GamвоA (G. J.), 1963. - Comprobacíon de Rhodnius prolixus extradomiciliario en Venezuela (comunicacíon preliminar). Bol. Of. Sanit. Panam., LIV, 1, 18-25.

Gomez Nunez (J. C), 1964. - Communication personnelle.

JiMeNEZ (J. C.), 1964. - Informe sobre la campaña contra la enfermedad de Chagas en el Estado Lara llevada a cabo, por el servicio de Endemias Rurales de la Zona VI. Folleto publicado por la Dirección de Malariología y Saneamiento Ambiental. División de Endemias Rurales, 14.

Langeron (M.), 1949. - Précis de Microscopie, Masson, Paris.

MAYeR (H.F.), 1961. - Infección experimental con Trypanosoma cruzi por vía digestiva. An. Inst. Med. Regional, 5 (3), 43-48, y en Rev. Fac. C. Vet. de la Plata, III, 9, 411-415.

Mayer (H. E.) et AlCaraz (I.), 1954. - Investigaciones sobre esquizotripanosis en perros y gatos de la zona suburbana de resistencia. Ann. Inst. Med. Regional, 4 (1) ; 9-17.

Pedreira de Freitas (J. L.), 1961. - Diagnóstico de laboratorio de molestias de Chagas. Bol. Of. Sanit. Panam, LI, 5, 429-438.

Pifano (C. F.), 1954. - El diagnóstico parasitológico de la enfermedad de Chagas en fase crónica. Estudio comparativo entre la gota gruesa y el xenodiagnóstico, el hemocultivo y las inoculaciones experimentales en animales sensibles. Arch. Venez Pat. Trop. y Paras. Médica, II, 2, 121-157.

-, 1960. - Algunos aspectos de la enfermedad de Chagas en Venezuela. Arch. Venez. Med. Trop. y Paras. Médica, III, 2, 73-101.

-, 1960. - La enfermedad de Chagas y sus problemas. Arch. Venez. Pat. Trop. y Paras. Médica, III, 2, 101-107.

Rodriguez Cabrera (C.) et Merino (E.), 1964. - Procolos de los estudios anatomopatológicos.

Romana (C.). 1963. - Enfermedad de Chagas, I Volumen.

TALICE (R. V.), 1944. - Enfermedades parasitarias del hombre y parásitos de interés médico, tome I, Montevideo.

Thomson (J. G.) et LAMBorn (W. A.), 1934. - a) Mechanical transmissión of trypanosomiasis, leishmaniasis, and yaws through the agency of non-biting haematophagous flies. Preliminary notes on experiments. Brit. Med. J. (3845), Sep. 15, 506-509.

Torrico (R. A.), 1950. - Conocimientos actuales sobre la enfermedad de Chagas en Bolivia, Bol. Of. Sanit. Panam., XXIX, 827-841.

Vergani (F.), 1952. Estudio sobre la vección de Trypanosomas por medio de Dipteros no vulnerantes. Bol. Inst. Inv. Vet., IV, 20, 657-672.

[Centro Experimental de Estudios Superiores, Barquisimeto (Venezuela)] 